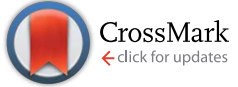

Cite this: RSC Adv., 2016, 6, 31131

Received 5th February 2016

Accepted 20th March 2016

DOI: $10.1039 / c 6 r a 03373 c$

www.rsc.org/advances

\title{
Enhanced sensitivity of quartz tuning fork sensors using electrospun polymer wires $\dagger$
}

\author{
Wuseok Kim, $\$$ Minhyuk Yun, $\$$ Seongjae Lee and Sangmin Jeon* \\ A polymethylmethacrylate (PMMA) nanowire was prepared by electrospinning and attached to the two \\ prongs of a quartz tuning fork (QTF) to form a polymer bridge. The attachment of the wire increased the \\ resonance frequency due to an increase in the stiffness of the QTF. The resonance frequency of the \\ PMMA wire-coated QTF decreased upon exposure to water vapor, which was attributed to a decrease in \\ the modulus of the wire induced by the adsorption of moisture. The sensitivity of the PMMA wire-coated \\ QTF was enhanced by increasing the number of PMMA wires on the QTF without affecting the response \\ time of the sensor.
}

\section{Introduction}

A quartz tuning fork (QTF) has a unique geometry comprising two vibrating prongs separated by a specific gap. The symmetry of the two prongs reduces the number of possible vibration modes, which endows the QTF with a good quality factor. ${ }^{1}$ The high stability and low power consumption of QTFs allow them to be used in a wide range of applications, including in precision watches ${ }^{2}$ and force sensors. ${ }^{3,4}$ In addition to these conventional applications, there have been attempts to use QTFs as versatile gas sensors because the resonance frequency of a QTF decreases with increased mass loading. ${ }^{5,6}$ However, the poor mass sensitivity of a typical $32.768 \mathrm{kHz}$ QTF $\left(\sim 50 \mathrm{ng} \mathrm{Hz}{ }^{-1}\right)$ limits its broader applicability in gas sensors.

Tao et al. addressed this problem by producing a polymer wire using a tip-drawing method and attaching the polymer wire to the two prongs of a QTF. ${ }^{7}$ In this configuration, the polymer wire is stretched and compressed by the two vibrating prongs; the change in the mechanical force of the polymer wire due to gas adsorption is easily measured from the change in the resonance frequency. ${ }^{8-14}$ The results showed that the performance of the QTF (i.e., the response time) can be improved by reducing the diameter of the attached polymer wire. However, it was difficult to produce a thin polymer wire using the tipdrawing method; thus, they reduced the diameter of a small portion of the wire from $15 \mu \mathrm{m}$ to $0.5 \mu \mathrm{m}$ using a focused ion beam under vacuum. Furthermore, the polymer wire produced by the tip-drawing method possessed an uneven thickness

Department of Chemical Engineering, Pohang University of Science and Technology (POSTECH), 77 Cheongam-Ro, Nam-Gu, Pohang, Gyeongbuk, Korea. E-mail: jeons@ postech.ac. $\mathrm{kr}$

$\dagger$ Electronic supplementary information (ESI) available. See DOI: 10.1039/c6ra03373c

\$ These authors contributed equally to this work. because the diameter of the polymer wire decreased as the wire was stretched.

In this study, we solved these problems by adopting an electrospinning method that utilizes electrical forces to produce polymer wires. Electrospinning has attracted considerable attention over the last decade due to its unique capability to produce a wide range of polymer wires in the submicron range. ${ }^{15-17}$ We fabricated thin polymethylmethacrylate (PMMA) wires using electrospinning and examined the PMMA wirecoated QTF as a moisture sensor. Upon exposure to water vapor, the resonance frequency of the PMMA wire-coated QTF decreased due to the decrease in the modulus of the PMMA wire, which was induced by the adsorption of moisture. Increasing the number of polymer wires improved the sensitivity of the sensor without affecting the sensor's response time.

\section{Experimental}

\section{Materials}

PMMA (molecular weight $(\mathrm{Mw})=350000 \mathrm{~g} \mathrm{~mol}^{-1}$ ) was purchased from Aldrich (Saint Louis, MO). Dimethylformamide (DMF) and tetrahydrofuran (THF) were obtained from Aldrich (Saint Louis, MO) and used without further purification. QTFs with widths of $250 \mu \mathrm{m}$, thicknesses of $600 \mu \mathrm{m}$, and lengths of $3400 \mu \mathrm{m}$ were purchased from Sunny Inc. (Chungju, Korea). The resonance frequency and spring constant of the bare QTF were $32.76 \mathrm{kHz}$ and $13 \mathrm{kN} \mathrm{m}^{-1}$, respectively.

\section{Instrumental setup for the electrospinning of PMMA wires}

Fig. 1 shows the home-built electrospinning instrument setup used in this study. A high-voltage power supply and disposable plastic syringe were purchased from Matsusada (Shiga, Japan) and Henke-Sass Wolf (Tuttlingen, Germany), respectively. A metal nozzle with an inner diameter of $330 \mu \mathrm{m}$ was purchased from NanoNC (Seoul, Korea). A 10 wt\% solution of PMMA in 

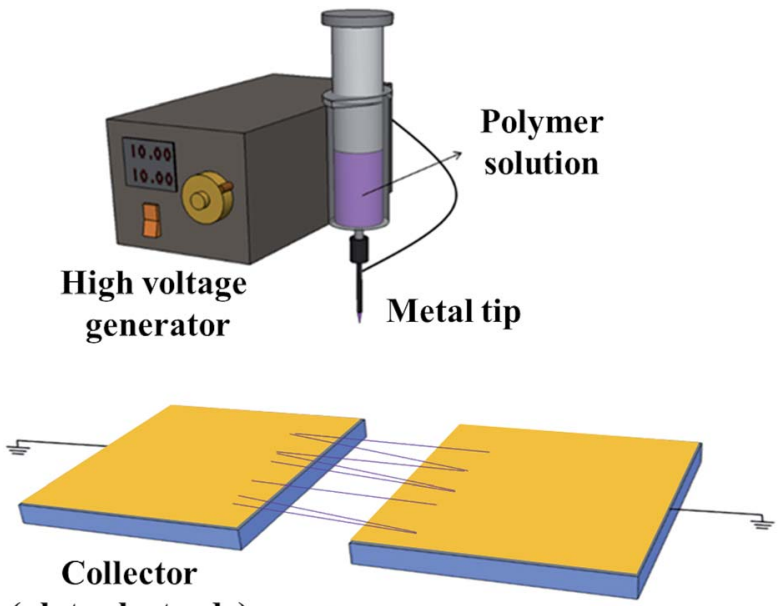

(plate electrode)

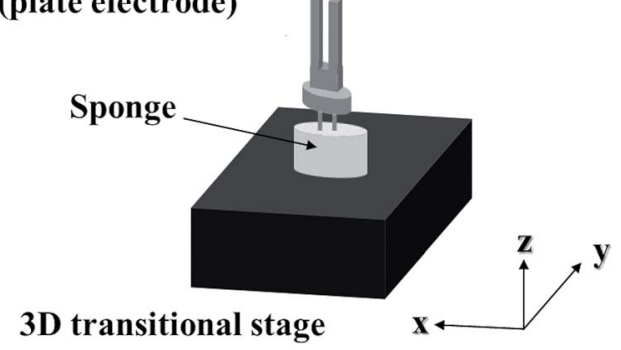

Fig. 1 Schematic of the instrumental setup for preparing PMMA wirecoated QTFs.

DMF was loaded into the $10 \mathrm{~mL}$ plastic syringe, and a potential of $15 \mathrm{kV}$ was applied to the nozzle to produce PMMA wires. The distance between the nozzle and the collector was fixed at $15 \mathrm{~cm}$. The PMMA wires were electrospun across the two separated gold-coated silicon wafers to obtain freestanding wires. ${ }^{18}$ The suspended wire was then transferred onto a QTF using a three-dimensional translational stage with an optical microscope. Multiple wire-coated QTF was obtained by repeating the process. A small amount of THF was dropped onto the prongs of the QTF to glue the wire. The PMMA wire-coated QTF was dried in a vacuum oven at $60{ }^{\circ} \mathrm{C}$ for $12 \mathrm{~h}$ to remove residual stress in the PMMA wire.

\section{Quartz tuning forks}

The resonance frequencies of QTFs were measured using a home-built PC-based conductance measurement system comprising a digitizer (NI PXI-5114), a multiplexer (NI PXI2593), and a function generator (NI PXI-5406), as previously reported. ${ }^{13}$ Briefly, the conductance spectra were obtained by measuring the admittance and phase of the QTF and fit with Lorentzian curves to calculate the resonance frequency in situ upon adsorption of moisture. Wet nitrogen was produced by passing dry nitrogen through a gas bubbler containing water. The wet and dry nitrogen streams were combined and injected into the flow cell. The flow rate of each gas stream was controlled using a mass flow controller (Brooks Instrument, MA); the total flow rate was fixed at $100 \mathrm{~mL} \mathrm{~min}{ }^{-1}$. The relative humidity was measured using a commercial humidity sensor
(Pico Technology, England), and all experiments were conducted at room temperature.

\section{Results and discussion}

Fig. 2(a) shows a scanning electron microscopy (SEM) image of the electrospun PMMA wires. The average wire diameter is $462 \mathrm{~nm} \pm 65 \mathrm{~nm}$, which is much thinner than that of the polymer wire produced by the tip-drawing method $(\sim 15 \mu \mathrm{m}) .{ }^{9}$ Fig. 2(b) shows optical microscopy images of the prongs of QTFs coated with different numbers of PMMA wires: bare QTF

(a)

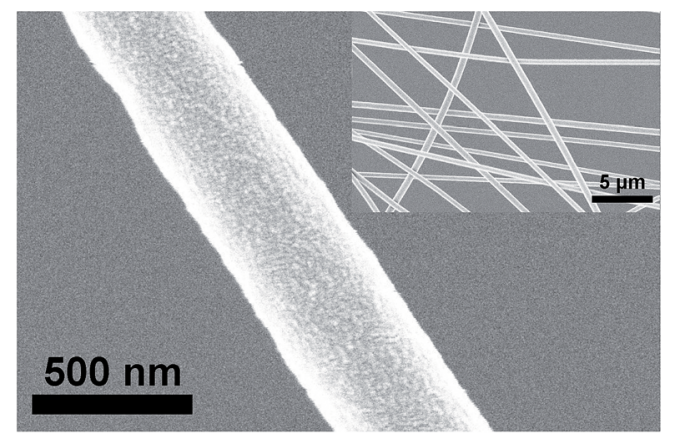

(b)

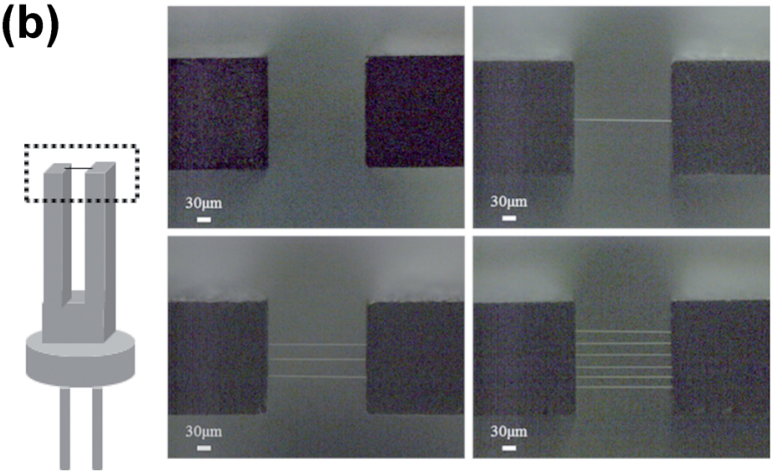

(c)

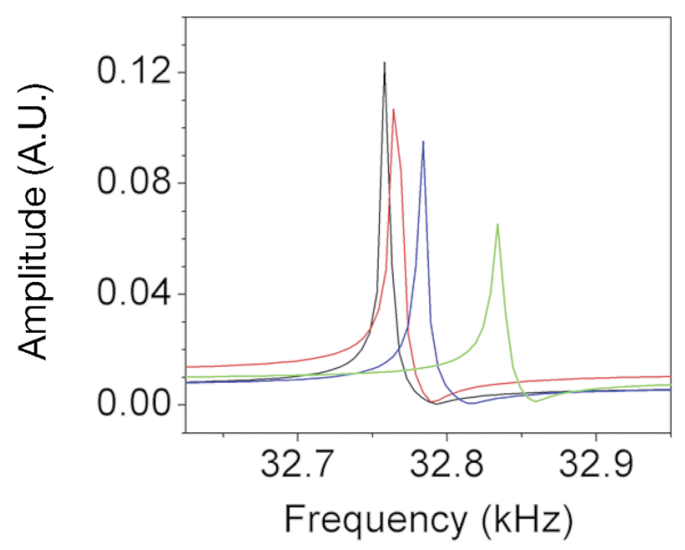

Fig. 2 (a) SEM image of an electrospun PMMA wire. The inset shows a SEM image of PMMA wires at reduced scale. (b) Optical microscopy images of the bare QTF, QTF1, QTF3, and QTF6. (c) The resonance frequency peaks before and after attaching the PMMA wire: bare QTF (black), QTF1 (red), QTF3 (blue), and QTF6 (green). 
(no wire), QTF1 (one wire), QTF3 (three wires), and QTF6 (six wires). The distance between the two prongs was $250 \mu \mathrm{m}$, and each wire was placed on the prongs of the QTF with a spacing of 20-30 $\mu \mathrm{m}$. Fig. 2(c) shows the resonance frequency peaks before and after attaching the PMMA wire. The resonance frequency of the bare QTF (32.76 kHz) increased to $32.77 \mathrm{kHz}$ (QTF1), 32.84 $\mathrm{kHz}$ (QTF3), and $32.89 \mathrm{kHz}$ (QTF6) after the attachment of the PMMA wires. The change in the resonance frequency $(\Delta f)$ of a QTF is affected by the change in the mass loading $(\Delta m)$ and in the effective stiffness $(\Delta k)$ as follows: ${ }^{19}$

$$
\Delta f=\left(\frac{f_{0}}{2}\right)\left(\frac{\Delta k}{k_{0}}-\frac{\Delta m}{m_{0}}\right) \approx \frac{f_{0}}{2 k_{0}} \Delta k
$$

where $f_{0}, k_{0}$, and $m_{0}$ are the resonance frequency, spring constant, and mass of the bare QTF, respectively. According to eqn (1), the resonance frequency should decrease because the increase in the number of PMMA wires increases the mass loading on the QTF. However, the resonance frequency of the PMMA wire-coated QTF was higher than that of the bare QTF and increased with an increase in the number of attached wires, indicating that the mass effects were negligible compared to the stiffness effects. A control experiment was conducted wherein the resonance frequency of the PMMA wire-coated QTF was measured after cutting the wire using a razor blade. The resonance frequency of the QTF with the PMMA wire residue was nearly identical to that of the bare QTF. Because the mass of a $1 \mu \mathrm{m}$-thick PMMA wire $(\sim 0.6 \mathrm{ng})$ is less than that of a QTF $(\sim 2.7 \mathrm{mg})$, the decrease in frequency due to the addition of a PMMA wire onto a bare QTF is only $0.004 \mathrm{~Hz}$, as calculated by eqn (1), confirming that the mass effects are negligible.

The influence of the number of attached PMMA wires on the sensitivity of the QTF was examined by exposing the QTF to a series of water vapor concentrations. Fig. 3 shows the timedependent variations in the resonance frequencies of the bare QTF, QTF1, QTF3, and QTF6. The concentrations of water vapor

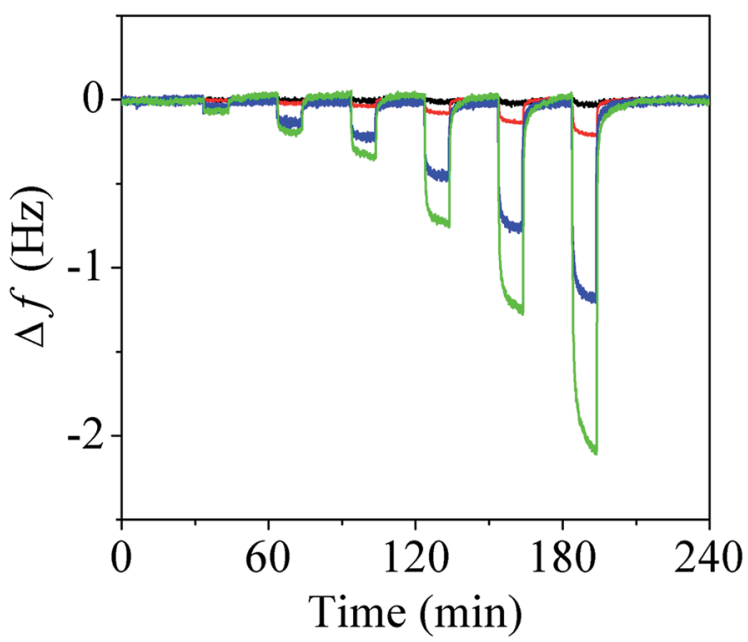

Fig. 3 Time-dependent variations in the resonance frequencies of the bare QTF (black), QTF1 (red), QTF3 (blue), and QTF6 (green) upon exposure to water vapor. The water vapor concentration was sequentially varied as follows: $8 \%$ (dry nitrogen), $11 \%, 15 \%, 18 \%, 26 \%$, $33 \%$, and $40 \%$. were sequentially changed as follows: $8 \%$ (dry nitrogen from a nitrogen cylinder) $\rightarrow 11 \% \rightarrow 15 \% \rightarrow 18 \% \rightarrow 26 \% \rightarrow 33 \% \rightarrow$ $40 \%$. After $10 \mathrm{~min}$ of exposure to water vapor, the flow cell was rinsed with dry nitrogen for $20 \mathrm{~min}$ to remove the water vapor from the PMMA wire. The recovery of the resonance frequency to the original value after rinsing confirms that the adsorption of water vapor onto the PMMA wire is reversible. Further, the results were reproducible for $>100$ repeated measurements, which indicates that the PMMA wire coating is very stable. The changes in the resonance frequency of the bare QTF were negligible, indicating that the bare QTF is insensitive to the adsorption of water vapor. However, the resonance frequencies of the PMMA wire-coated QTFs decrease with increasing humidity because the absorption of water vapor decreased the stiffness of the PMMA wire..$^{20}$ The response time of a sensor is defined as the time required for the frequency to decrease by $63.2 \%$. The response times of the QTFs were almost identical ( $\sim 16 \mathrm{~s}$ ) regardless of the number of PMMA wires (Fig. S1; ESI $\dagger$ ).

Fig. 4 shows the changes in the resonance frequencies of the bare QTF, QTF1, QTF3, and QTF6 as functions of relative humidity. The QTFs with greater numbers of wires exhibited larger changes in resonance frequency upon exposure to the same concentration of water vapor because $\Delta k$ in eqn (1) is proportional to the number of wires. Upon exposure to $40 \%$ humidity, the changes in the resonance frequencies of QTF1, QTF3, and QTF6 were found to be 10.3-, 23.0-, and 40.7-times that of the bare QTF, respectively. This result implies that the sensitivity of the PMMA wire-coated QTF can be enhanced by increasing the number of PMMA wires. The detection limit of QTF6 was found to be 8.1\% (water concentration of dry nitrogen from a nitrogen cylinder is $8.0 \%$ ). However, a control experiment showed that the attachment of random PMMA wires increased the noise level of the resonance frequency due to

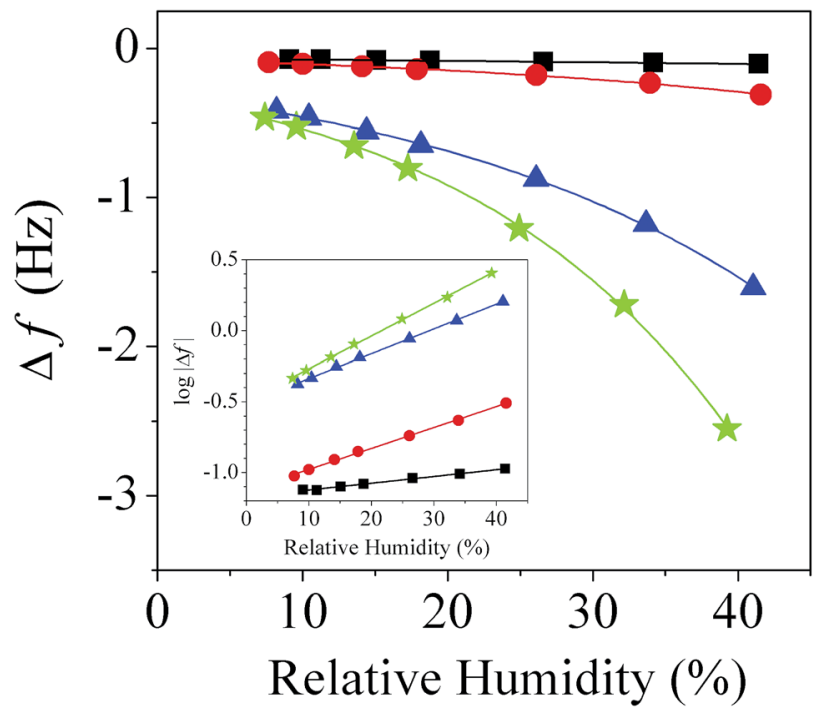

Fig. 4 The changes in the resonance frequencies of the bare QTF (black square), QTF1 (red circle), QTF3 (blue triangle), and QTF6 (green star) are shown as functions of relative humidity. Inset shows the logarithms of the resonance frequency changes as a function of relative humidity. 
asymmetric vibration damping (Fig. S2 $\dagger$ ). Inset shows that the logarithms of the resonance frequency changes are linear with respect to relative humidity.

\section{Conclusions}

In summary, we prepared PMMA wires using electrospinning and investigated the PMMA wire-coated QTF as a humidity sensor. Upon exposure to water vapor, the resonance frequency of the PMMA wire-coated QTF decreased due to the decrease in the modulus of the PMMA wire, which was induced by the adsorption of moisture. Increasing the number of PMMA wires enhanced the sensitivity of the QTF without affecting the response time of the sensor. Controlled alignment and patterning of thinner polymer wires on the QTF could be achieved by optimizing the electrospinning conditions and by utilizing an electrohydrodynamic nanowire printer. ${ }^{21}$

\section{Acknowledgements}

This work was supported by the National Research Foundation of Korea (NRF) grant funded by the Korea government (MEST) (NRF-2014R1A2A2A01007027).

\section{References}

1 J. M. Friedt and E. Carry, Am. J. Phys., 2007, 75, 415-422. 2 H. Yoda, H. Ikeda and Y. Yamabe, 26th Annual Symposium on Frequency Control. 1972, IEEE, New York, 1972.

3 H. Edwards, L. Taylor, W. Duncan and A. J. Melmed, J. Appl. Phys., 1997, 82, 980-984.

4 F. J. Giessibl, Appl. Phys. Lett., 1998, 73, 3956-3958.

5 X. Zhou, T. Jiang, J. Zhang, X. Wang and Z. Zhu, Sens. Actuators, B, 2007, 123, 299-305.
6 K. Waszczuk, G. Gula, M. Swiatkowski, J. Olszewski, W. Herwich, Z. Drulis-Kawa, J. Gutowicz and T. Gotszalk, Sens. Actuators, B, 2012, 170, 7-12.

7 S. Boussaad and N. J. Tao, Nano Lett., 2003, 3, 1173-1176.

8 S. Guan, Anal. Chem., 2003, 75, 4551-4557.

9 M. Ren, E. S. Forzani and N. Tao, Anal. Chem., 2005, 77, 2700-2707.

10 R. A. Iglesias, F. Tsow, R. Wang, E. S. Forzani and N. Tao, Anal. Chem., 2009, 81, 8930-8935.

11 R. Wang, F. Tsow, X. Zhang, J. H. Peng, E. S. Forzani, Y. Chen, J. C. Crittenden, H. Destaillats and N. Tao, Sensors, 2009, 9, 5655-5663.

12 M. Yun, S. Lee, C. Yim, N. Jung, T. Thundat and S. Jeon, Appl. Phys. Lett., 2013, 103, 053109.

13 S. Lee, M. Yun and S. Jeon, Appl. Phys. Lett., 2014, 104, 053506.

14 M. Yun, E. Lee, K. Cho and S. Jeon, Appl. Phys. Lett., 2014, 105, 073116.

15 Z. M. Huang, Y. Z. Zhang, M. Kotaki and S. Ramakrishna, Compos. Sci. Technol., 2003, 63, 2223-2253.

16 A. Greiner and J. H. Wendorff, Angew. Chem., 2007, 46, 56705703.

17 G. Collins, J. Federici, Y. Imura and L. H. Catalani, J. Appl. Phys., 2012, 111, 044701.

18 D. Li, Y. L. Wang and Y. N. Xia, Nano Lett., 2003, 3, 11671171.

19 A. Rai, F. Tsow, S. Nassirpour, J. Bankers, M. Spinatsch, M. P. He, E. Forzani and N. J. Tao, Sens. Actuators, B, 2009, 140, 490-499.

20 C. Ishiyama and Y. Higo, J. Polym. Sci., Part B: Polym. Phys., 2002, 40, 460-465.

21 S.-Y. Min, T.-S. Kim, B. J. Kim, H. Cho, Y.-Y. Noh, H. Yang, J. H. Cho and T.-W. Lee, Nat. Commun., 2013, 4, 1773. 